\section{CirCULATING Clones OF COMMUNITY-ACQUiRed MEthICILLIN-RESISTANT StaPHYLOCOCCUS AUREUS (CA- MrSa) In Western Australia In The Past 11 Years (2002-2013)}

\author{
A. M. Setiawan*
}

Faculty of Science and Technology, Islamic State University Maulana Malik Ibrahim, 65144, Malang, Indonesia
Article history

Received

7 July 2015

Received in revised form

3 November 2015

Accepted

3 March 2016

*Corresponding author drmalique@gmail.com

\section{Graphical abstract}
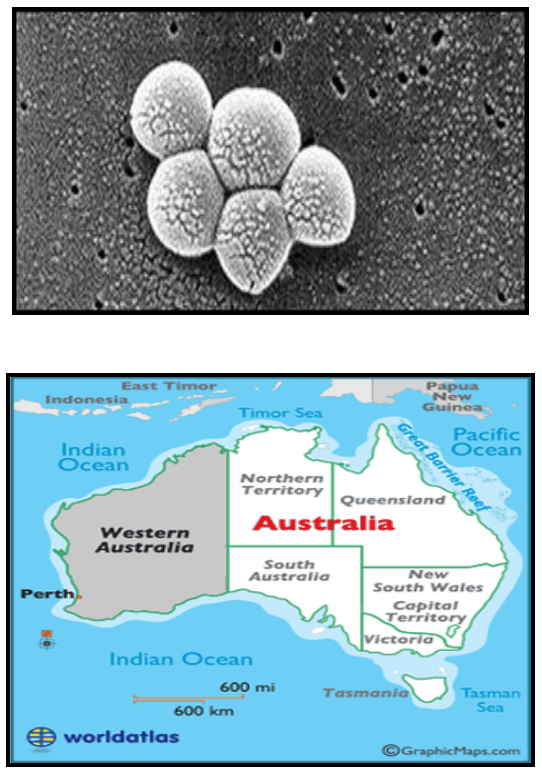

\begin{abstract}
Methicillin-resistant Staphylococcus aureus (MRSA) is one of the most complicated problems in infection control and disease prevention. Imported strains or group of similar strains called clones were introduced from other states within Australia and also from other countries. Western Australia (WA) MRSA clones known to be the most predominant clones circulating in Western Australia (WA). However, MRSA strains originally from other states and countries outside Australia (imported) have increased in number in the last decades. The aim of this study was to examine the epidemiology of CA MRSA clones in Western Australia for the past 11 year's period (2003-2013). There were 10 predominant clones of CA-MRSA circulating in WA with WA MRSA-1 $(32,82 \%)$, Queensland $(22,67 \%)$ and WA MRSA-2 $(22,67 \%)$ clones as the top three predominant clones. The percentage of PVL positive CA-MRSA clones such as Queensland (QId) clone has been increasing significantly $\left(R^{2}=0,67\right)$ from $2,38 \%$ in 2002 to $25,69 \%$ in 2013 . In contrast, the percentages of two major PVL negative clones, WA MRSA-1 and WA MRSA 2, have been decreasing nearly by half from $52,38 \%$ to $27,71 \%$ and $30,95 \%$ to $16,59 \%$ respectively in the last 11 years. PVL positive clones have been introduced in WA from other states and overseas in the last eleven years. Due to the virulence factor of PVL toxin, the emergence of PVL positive CA-MRSA should be considered as public health concerns.
\end{abstract}

Keywords: Building maintenance management, occupant satisfaction, school building

(C) 2016 Penerbit UTM Press. All rights reserved

\subsection{INTRODUCTION}

Staphylococcus aureus are the most common bacteria that can cause infections in all parts of human body such as in the skin, soft tissue, bloodstream, respiratory and urinary tract. Staphylococcus aureus can also be found in almost all geographic areas. Methicillin-resistant Staphylococcus aureus (MRSA) is one of the most complicated problems in infection control and disease prevention in hospital and health care setting (1). According to the pattern of antimicrobial resistance, there were two groups of MRSA circulated in Western Australia that can be classified as multi drug-resistant imported clones and less drug-resistant clones (WA MRSA) (2). Imported strains or group of similar strains called clones were introduced from other states within Australia and also from other countries. WA MRSA clones known to be the most predominant clones circulating in Western Australia (WA). However, MRSA strains originally from other states and countries outside WA (imported) has been increasing in number in the last decades (3).

Clonal types of CA MRSA circulated in Australia were quite diverse compare to other countries. In United States for example, USA300 MRSA clones 
counted at least $70 \%$ as the cause of skin and soft tissue infections (4). A multicenter study about genetic diversity among Community-Acquired (CA) MRSA in Europe showed USA300 clones were the predominant clones $(40 \%)$ circulated in several countries in Europe (5). In contrast, there were at least 59 clones of CA MRSA in Australia have been identified (6). The diversity of CA MRSA clones increasing in number over the last two decades. Most of the new clones were originally from overseas which imported from other countries and then established in Australia.

Generally, imported clones have higher virulence properties compared to MRSA clones originated from WA (WA MRSA). One of the virulence properties is called Pantone-Valentine Leukocidine (PVL). A PVL positive MRSA clones have emerged as a cause of both hospital-acquired (HA) and communityacquired (CA) infections in Western Australia (7). CA MRSA accounted for around $70 \%$ while HA MRSA 30 $\%$ from all MRSA infections in Australia. A significant increase of CA MRSA has been reported between 1998 and 2002 in Western Australia (3). In 2013, Kimberly region accounted having the highest CAMRSA colonisation rates in Western Australia $(2,176 / 100,000$ population) (8). The aim of this study was to examine the epidemiology of CA MRSA clones in Western Australia for the past 11 year's period (2003-2013)

\subsection{METHODOLOGY}

A systematic review was performed to identify eligible articles. The MEDLINE database from 2002 to 2013 was collected by combining words of "clones", "MRSA", "Methicillin-Resistant", "Staphylococcus aureus", "epidemiology" and "Western Australia". Only articles reporting the epidemiology of CA-MRSA clones in Western Australia were included.

\subsection{RESULTS AND DISCUSSION}

In the past 11 years, 9 studies were conducted to analysis the clones of CA-MRSA circulating in WA with a total 15.512 isolates. Among these isolates, there were 10 predominant clones of CA-MRSA circulating in WA as seen in Figure 1; WA MRSA-1 (32,82\%), Queensland (22,67 \%), WA MRSA-2 (22,67 \%), WA MRSA-3 (9,86 \%), WSPP MRSA (4,81\%), WA MRSA-121 $(1,85 \%)$, USA300 $(1,64 \%)$, Taiwan CA-MRSA $(0,72 \%)$, Bengal Bay $(0,61 \%)$ and WA MRSA-62 $(0,49 \%)$.

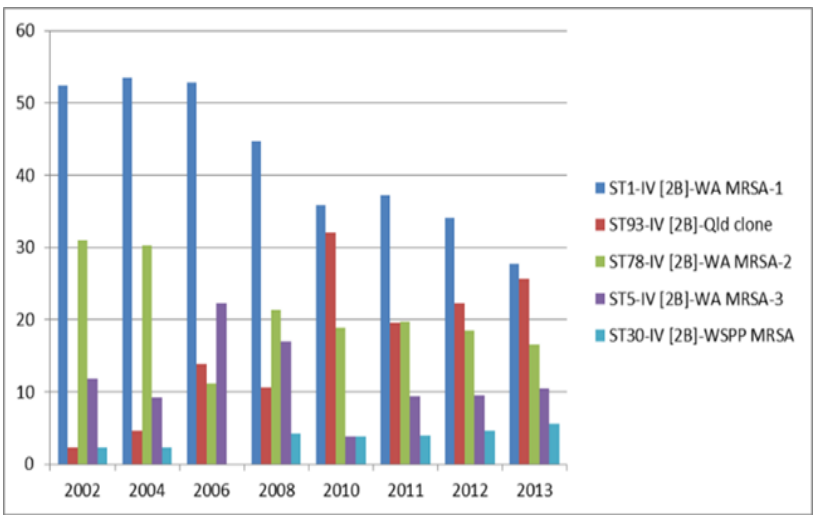

Figure 1 Percentages of five predominant MRSA clones circulated in WA from 2002 to 2013

Figure 2 shows the trend of 5 major clones of CAMRSA in WA in the past 11 years. The percentages of PVL positive CA-MRSA clones such as Queensland (QId) clone has been increasing significantly (R2 $=0,67)$ from $2,38 \%$ in 2002 to $25,69 \%$ in 2013 . In contrast, the percentages of two major PVL negative clones, WA MRSA-1 and WA MRSA 2, have been decreasing nearly by half from $52,38 \%$ to $27,71 \%$ and $30,95 \%$ to $16,59 \%$ respectively in the last 11 years (6-11). Of five predominant CA MRSA clones (n $=13.709$ isolates), There were 3 CA WA-MRSA and 2 imported CA MRSA identified as the top five predominant clones in the last 11 years. The average percentages of CA WA-MRSA and imported CA MRSA were $76,09 \%$ ( $n=9.446$ isolates) and $19,36 \%$ (n $=4.263$ isolates) respectively.

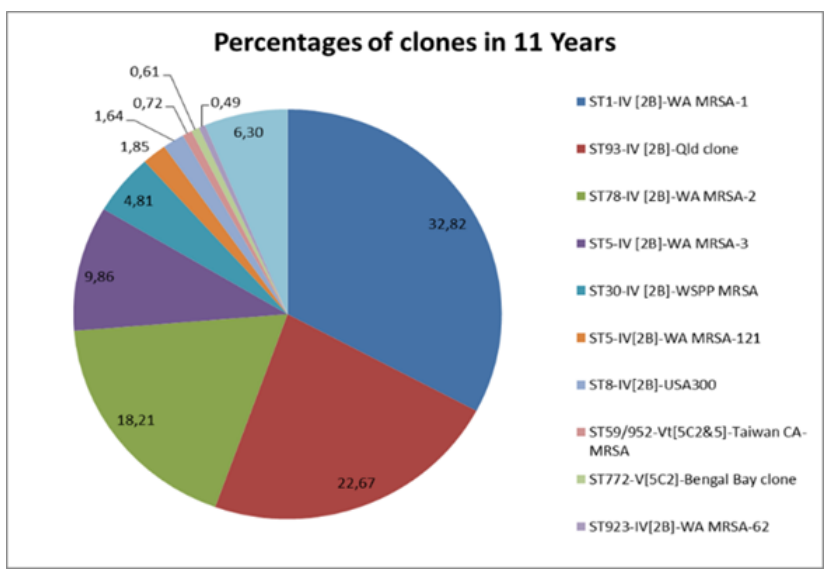

Figure 2 Average percentages of CA MRSA clones in WA within 11 years

Staphylococcus aureus can be found in the surface of human bodies and has the ability to develop antimicrobial resistance by acquiring resistance gene from other resistant strains. S. aureus resistance to methicillin were discovered not too long after methicillin being used in 1960. Since that time, several outbreaks have been reported around the 
world including in Australia in 1976. The MRSA strains that caused different outbreaks around the world were thought to be genetically different according to the geographical area where those particular strains have emerged. However, after the application of multilocus sequence typing (MLST) combined with Staphylococcal chromosome cassete mec (SCCmec) typing by Enright et al. in 2002, there were only several major MRSA clones known to be circulated around the world (12).

First MRSA cases in Western Australia were discovered in 1982 when there was an outbreak in a teaching hospital. The outbreak was occurred in Royal Perth Hospital after the admission of patient with MRSA infection from other states (13). The MRSA strain from this outbreak was categorized as imported strains and called as epidemic MRSA (EMRSA) because from the genetic analysis of the isolates showed that these strains were similar to the first MRSA strains isolated in Eastern Australia in 1976 called Eastern Australian MRSA (EA MRSA) (14).

MRSA colonisations have become a notifiable disease in WA since there were an MRSA outbreak in 1982. Following this, MRSA stewardship program has been activated to prevent imported strains of MRSA to become established in any hospital within WA. The policy require all patients who had been treated in any hospital outside WA in the previous 12 months should be screened for MRSA carriage $(13,14)$. This policy also require MRSA screening to all health care workers who have been working outside WA in the previous 12 months before working in WA health care services. This policy was very useful to prevent the establishment of epidemic MRSA strains in WA hospitals and other health care services (15).

In 1990, there was an increase in number of MRSA infections in Perth area. Most of the patients never been to any places outside WA. In fact, most of the patients were originally from Kimberly region of northern WA. The genetic analysis of the isolates revealed that this MRSA incidences was caused by new non-multidrug resistant MRSA strains and differ to the earlier strains (EA MRSA) (14). This finding can be considered as the early cases involving WA MRSA strains in WA. However, some WA MRSA strains then evolved and acquired multi-drug resistance plasmids which encode resistance properties to antimicrobials.

Community acquired MRSA can be found in any form of infections from minor skin and soft tissue infections to bacteremia with sepsis. There were no significant differences between CA MRSA and HA MRSA in term of severity of illness. In addition, CA MRSA clones were interestingly found to be the most predominant causes of HA MRSA bacteremia in some studies and it's even higher compare to HA MRSA clones itself (16). A screening study conducted by Dailey et al. (2005) revealed that WA MRSA were the predominant CA MRSA strains circulated in WA between 1998 and 2002. Overall, there were three CA WA MRSA clones (ST1-MRSA, ST129-MRSA and ST5MRSA) circulated in Western Australia during this period. However, there was a significant increase in the incidence of imported MRSA strains from 6,4\% notifications in 1998 to $24,4 \%$ in 2002 (3). Even though the number of WA MRSA strains incidences were increasing during this period, the ratio between WA MRSA strains to imported MRSA strains was decreased.

There were 9 epidemiological studies conducted in the last 11 years in WA which examining the diversity of CA MRSA clones. In total, 15.512 isolates of CA MRSA were examined using similar techniques and methods to identify the genetic properties of each isolates. Pulsed-field gel electrophoresis (PFGE), spa typing, multi locus sequence typing (MLST) and PCR test for detecting staphylococcal cassette chromosome mec (SCCmec) elements and PantonValentine Leukocidin (PVL) were used to determine the characteristic of each isolates and differentiate into different clones.

The results from this study show similar patterns with the previous studies. WA MRSA-1 clones remain the most predominant CA MRSA clones isolated in WA from 2002 to 2013. Of the top five predominant clones ( $n=13.709$ isolates) circulated in WA between 2002 and 2013, three CA WA-MRSA clones (ST1-MRSA, STI29-MRSA and ST5-MRSA) and two imported CA MRSA clones were identified (QId and WSPP MRSA clones). The average percentages of CA WA-MRSA and imported CA MRSA clones counted as many as $76,09 \%$ ( $n=9.446$ isolates) and 19,36\% ( $n=4.263$ isolates) respectively. Figure 3 provides the comparison of the percentages between CA WAMRSA clones and imported MRSA clones in the last 11 years. There were an increasing number of imported CA MRSA clones from 2002 (5,76\%) to 2013 (37,32\%). In contrast, the percentages of CA WA-MRSA clones have been decreased nearly by half from $95,23 \%$ in 2002 to become $54,72 \%$ in 2013.

WA MRSA clones known to be less drug-resistant clones compared to imported MRSA clones. Generally, WA MRSA clones were not carry the gene encoding Panton-Valentine leukocidin (PVL). PVL is a toxin that capable to create polymeric pores in leukocyte membrane (2). Most of PVL positive strains isolated from abscess and furunculoses were resistant to many antimicrobial drugs especially from $\beta$ Lactam groups. In contrast, PVL negative strains usually less resistant and easy to treat $(17,18)$.

The ratio between WA CA-MRSA and imported CAMRSA as seen in Figure 3 shows how the establishment of imported CA-MRSA has been changed in the last decades. It is poorly understood why the population of imported CA-MRSA are increasing. Regarding the acquisition of PVL encoding gene (PVL positive clones), all three WA CA-MRSA clones were PVL negative while all two imported CA-MRSA clones were PVL positive. It can be assume that the increase of imported CA-MRSA clones circulating in WA implicate to the increase of virulent MRSA strains population. 


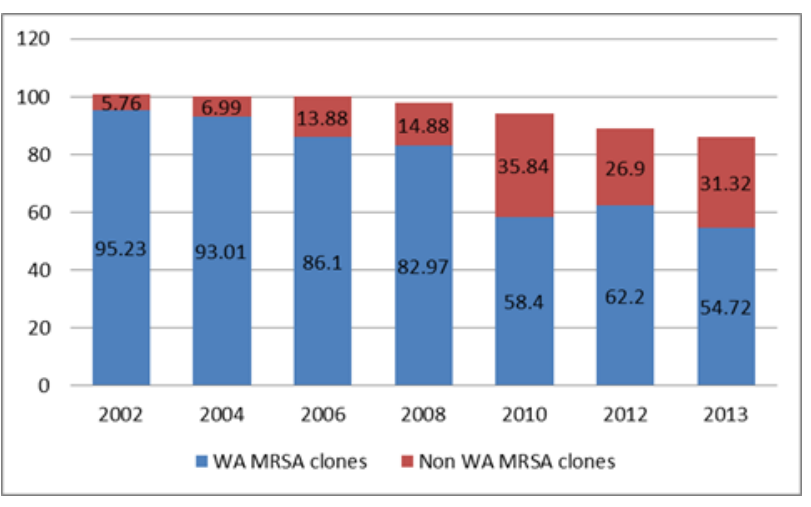

Figure 3 Percentages of WA and non-WA CA-MRSA clones circulated in WA from 2002 to 2013

\subsection{CONCLUSION}

In conclusion, WA CA-MRSA still become the most predominant clones circulating in WA in the last 11 years. There were an increasing number of imported CA MRSA clones circulated in WA. PVL positive, Queensland, MRSA clones were increasing in number of incidences in WA between 2002 and 2013. Most of WA MRSA clones were PVL negative while most of imported clones were PVL positive clones. PVL encoded gene could be transmitted from PVL positive clones to $\mathrm{PVL}$ negative clones and increase the virulence of WA MRSA clones. PVL proteins will increase the morbidity of MRSA colonization and infection in a patient as it cause leukocyte destructions and necrotic lesions. PVL positive clones have been introduced in WA from other states and overseas in the last eleven years. Due to the virulence factor of $P V L$ toxin, the emergence of $P V L$ positive CA-MRSA should be considered as public health concerns. MRSA stewardship policy could prevent the establishment of PVL positive CA MRSA clones in hospital and health care services. However, similar policy and program should be delivered to community in order to prevent the establishment of PVL positive MRSA clones in community.

\section{References}

[1] Diekema, D. J. et al. 2001. Survey Of Infections Due To Staphylococcus Species: Frequency Of Occurrence And Antimicrobial Susceptibility Of Isolates Collected in the United States, Canada, Latin America, Europe, and the Western Pacific region for the SENTRY Antimicrobial Surveillance Program, 1997-1999. Clinical Infectious Diseases: An Official Publication of The Infectious Diseases Society Of America. 32(2): S1 14-32.

[2] Monecke, S. et al. 2009. The Molecular Epidemiology And Evolution of The Panton-Valentine LeukocidinPositive, Methicillin-Resistant Staphylococcus Aureus Strain USA300 In Western Australia. Clinical Microbiology And Infection: The Official Publication Of The European Society Of Clinical Microbiology And Infectious Diseases. 15(8): 770-776.
[3] Dailey, L. et al. 2005. Methicillin-resistant Staphylococcus aureus, Western Australia. Emerging Infectious Diseases. 11 (10): 1584-1590.

[4] Tong, S.Y.C. et al. 2008. Global Implications of the Emergence of Community-Associated MethicillinResistant Staphylococcus aureus in Indigenous Populations. Clinical Infectious Diseases. 46(12): 18711878.

[5] Rolo, J. et al. 2012. High Genetic Diversity among Community-Associated Staphylococcus aureus in Europe: Results from a Multicenter Study. PLOS ONE. 7(4): 1-10.

[6] Coombs, G. W. et al. 2010. Differentiation of Clonal Complex 59 Community-Associated Methicillin-Resistant Staphylococcus aureus in Western Australia. Antimicrobial Agents And Chemotherapy. 54(5): 19141921.

[7] Turnidge, J. D. et al. 2013. Australian Group on Antimicrobial Resistance Community-onset Gramnegative Surveillance Program annual report, 2010. Communicable Diseases Intelligence Quarterly Report. 37(3): E219-23.

[8] Turnidge, J. D. et al. 2014. Australian Group on Antimicrobial Resistance Community-onset Gramnegative Surveillance Program Annual Report, 2012. Communicable Diseases Intelligence Quarterly Report. 38(1): E54-E8.

[9] Combs, G. W., Pearson, J. C., Christiansen, K. 2011 Western Australian Methicillin-resistant Staphylococcus aureus (MRSA) and Vancomycin Resistant Enterococcus (VRE) Epidemiology and Typing Report 2010-201 1. Perth: Australian Collaborating Centre for Enterococcus and Staphylococcus Species (ACCESS) Typing and Research

[10] Combs, G. W., Pearson, J. C., Robinson O. 2013. Western Australian Methicillin-resistant Staphylococcus aureus (MRSA) and Vancomycin Resistant Enterococcus (VRE) Epidemiology and Typing Report 2012-2013. Perth: Australian Collaborating Centre for Enterococcus and Staphylococcus Species (ACCESS) Typing and Research

[11] Combs, G. W. et al. 2012. Western Australian Methicillinresistant Staphylococcus aureus (MRSA) and Vancomycin Resistant Enterococcus (VRE) Epidemiology and Typing Report 2011-2012. Perth: Australian Collaborating Centre for Enterococcus and Staphylococcus Species (ACCESS) Typing and Research.

[12] Enright, M. C. et al. 2009. The Evolutionary History Of Methicillin-Resistant Staphylococcus aureus (MRSA). Proc Natl Acad Sci USA. 99(11): 7687-7692.

[13] Udo, E. E., Pearman J. F., Grubb, W. B. 1993. Genetic Analysis Of Community Isolates Of Methicillin-Resistant Staphylococcus aureus in Western Australia. Journal of Hospital Infection. 25(2): 97-108.

[14] Riley, T. V., Rouse, I. L. 1995. Methicillin-resistant Staphylococcus aureus in Western Australia, 1983-1992. The Journal of Hospital Infection. 29(3):177-188.

[15] Coombs, G. W. et al. 2007. Controlling a Multicenter Outbreak Involving the New York/Japan Methicillin-Resistant Staphylococcus aureus Clone. Infection Control and Hospital Epidemiology. 28(7): 845852.

[16] Robinson, J. O. et al. 2009. Community-associated Versus Healthcare-Associated Methicillin-Resistant Staphylococcus aureus bacteremia: a 10-year Retrospective Review. Eur J Clin Microbiol Infect Dis. 28(4): 353-361.

[17] Takizawa, Y. et al. 2005. A Panton-Valentine leucocidin (PVL)-positive Community-Acquired Methicillin-Resistant Staphylococcus Aureus (MRSA) Strain, Another Such Strain Carrying A Multiple-Drug Resistance Plasmid, And Other More-Typical PVL-Negative MRSA Strains Found in Japan. 43(7): 3356-3363. 
[18] Vourli, S. et al. 2009. High Rates Of CommunityAcquired, Panton-Valentine Leukocidin (PVL)-Positive
Methicillin-Resistant S. Aureus (MRSA) Infections In Adult Outpatients in Greece. 14(2): 19089. 